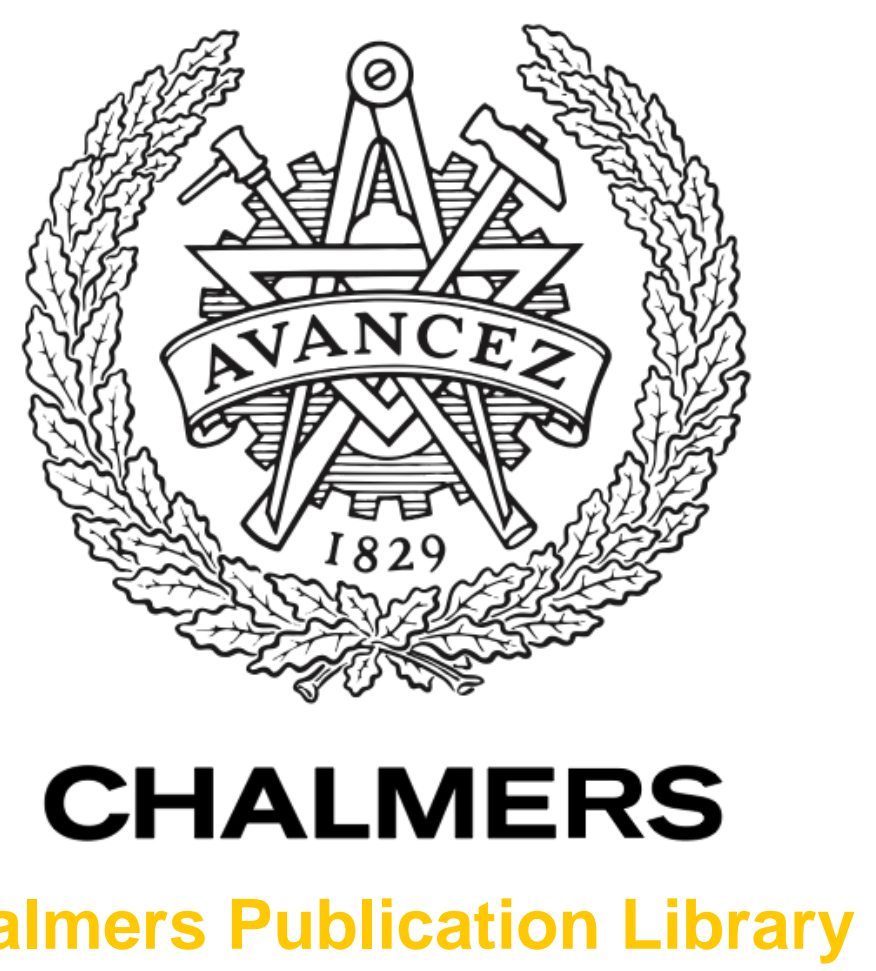

\title{
Resource Sharing and Power Allocation for D2D-based Safety-Critical V2X Communications
}

This document has been downloaded from Chalmers Publication Library $(\mathrm{CPL})$. It is the author's version of a work that was accepted for publication in:

\section{IEEE ICC 2015 Workshop}

Citation for the published paper:

Wanlu, S. ; Yuan, D. ; Ström, E. et al. (2015) "Resource Sharing and Power Allocation for D2D-based Safety-Critical V2X Communications". IEEE ICC 2015 Workshop

Downloaded from: http://publications.lib.chalmers.se/publication/214691

Notice: Changes introduced as a result of publishing processes such as copy-editing and formatting may not be reflected in this document. For a definitive version of this work, please refer to the published source. Please note that access to the published version might require a subscription. 


\title{
Resource Sharing and Power Allocation for D2D-based Safety-Critical V2X Communications
}

\author{
Wanlu Sun ${ }^{1}$, Di Yuan ${ }^{2,3}$, Erik G. Ström ${ }^{1}$, and Fredrik Brännström ${ }^{1}$ \\ ${ }^{1}$ Department of Signals and Systems, Chalmers University of Technology, 41296 Gothenburg, Sweden \\ ${ }^{2}$ Department of Science and Technology, Linköping University, 60174 Norrköping, Sweden \\ ${ }^{3}$ Institute for Systems Research, University of Maryland, College Park, MD, USA \\ wanlu@chalmers.se,di.yuan@liu.se, \{fredrik.brannstrom,erik.strom\}@chalmers.se
}

\begin{abstract}
Deploying direct device-to-device (D2D) links is considered an enabler for V2X applications, with intra-cell interference and stringent latency and reliability requirements as challenging issues. We investigate the radio resource management problem for D2D-based safety-critical V2X communications. Firstly, we analyze and transform the V2X latency and reliability requirements into mathematical forms that are computable using only slowly varying channel information. Secondly, we propose a problem formulation fulfilling the requirements of $\mathrm{V} 2 \mathrm{X}$, where resource sharing can take place not only between vehicles and cellular users but also among different vehicles. Moreover, a Resource Block Sharing and Power Allocation (RBSPA) algorithm is proposed to solve this problem. Finally, simulations are presented that indicate promising performance of the proposed RBSPA scheme.
\end{abstract}

\section{INTRODUCTION}

\section{A. Motivation}

By recent market analysis, vehicles become the third type of fastest growing connected devices after smart phones and tablets [1]. As a result, communications in moving networks are attracting great interests. In this context, V2X communication plays a crucial role since it enables reliable and lowlatency services such as traffic safety [2]. Here "V2X" is a term that collectively refers to vehicle-to-vehicle (V2V), vehicleto-infrastructure (V2I), and vehicle-to-device (V2D). In this work, we focus on safety-critical V2X applications, which usually have a strongly localized nature. V2X applications come with real-time requirements and stringent requirements on reliability and access availability. For instance, the European Union METIS project [2] considers a maximum end-toend delay of $5 \mathrm{~ms}$ and transmission reliability of $99.999 \%$.

A related trend is the consideration of device-to-device (D2D) communications. In a D2D underlaying cellular infrastructure, two physically close user equipment (UE) devices can directly communicate with each other by sharing the same resources used by regular cellular UEs (C-UEs), with the benefits of proximity gain, reuse gain, and hop gain [3].

The analysis in [4], [5] led to two conclusions for V2X and D2D. First, current legacy solutions for V2X communication, e.g., ad-hoc communication over the 802.11p standard and backend-based communication cellular networks, are not able to satisfy the quality of service (QoS) requirements of

This work has been supported in part by the Swedish Research Council project 2011-5824. Part of this work has been performed in the framework of the FP7 project ICT-317669 METIS, which is partly funded by the EU. The authors would like to acknowledge the contributions of their colleagues in METIS, although the views expressed are those of the authors and do not necessarily represent the project. safety-critical V2X applications, particularly in dense traffic. Second, by the similarities between the requirements on V2X communication and the characteristics of direct D2D, the latter is a promising approach for implementing the former.

\section{B. State of the Art}

Extensive research has been carried out in the context of conventional D2D systems, where one of the main challenges is the interference between the primary cellular network and the D2D underlay. To cope with this interference situation, one crucial issue is the radio resource management (RRM) strategy. For this line of research, the interested readers are referred to the surveys in [3], [6], and the references therein.

There are three major limitations in most of the existing D2D works for our target application of safety-critical V2X. Firstly, the performance objective has been typically to maximize the sum rate and prioritize cellular links [7], [8]. Thus the D2D underlay is considered opportunistic as their interference to cellular links is controlled to be acceptable levels. As a result, schemes for traditional D2D systems cannot work for V2X with small message payload but very strict requirements on latency and reliability. Secondly, the majority of the literature assumes that the eNB is aware of the full instantaneous channel state information (CSI) of all the cellular and D2D links; this assumption is probably too optimistic for fast moving D2D-based V2X communication. Last but not least, most of previous studies consider the setup that any RB of C-UEs can be shared by at most one D2D link [5], [7]. In fact, allowing multiple and concurrent D2D transmissions on the same RB and assuring the links' quality will not only improve spectrum efficiency by higher number of D2D links, but also may lead to less interference to CUEs due to spatial reuse. Recently, the authors of [8]-[10] considered the coexistence of multiple D2D links on the same $\mathrm{RB}$; however the setup in [8]-[10] does not apply D2D to V2X communications.

When it comes to using a D2D underlay for V2X communications, to the best of our knowledge, only few studies [1], [2], [4], [5] have been conducted. In particular, we have in [5] proposed a two-stage resource block (RB) allocation and power control scheme for D2D-based V2V communication, considering QoS requirements of both vehicular UEs (V-UEs) and $\mathrm{C}-\mathrm{UEs}$, with orthogonal resource allocation for $\mathrm{V}$-UEs.

\section{Contributions}

In this work, a RB Sharing and Power Allocation (RBSPA) algorithm is proposed for D2D-based safety-critical V2X communication. The main contributions are as follows. 
- We propose a method to transform the strict latency and reliability requirements of V2X communication into optimization constraints that use only slowly varying CSI.

- Allowing non-orthogonal access for V-UEs, we formulate an optimization problem for allocating multiple RBs and transmit power to a set of C-UEs and V-UEs.

- Due to the problem's NP-hardness, we propose a heuristic two-stage RBSPA scheme which is a long-term RRM method requiring only slowly varying CSI at the eNB.

\section{Preliminaries}

\section{A. Notation}

Sets are denoted by calligraphic letters, e.g., $\mathcal{X}$, with $|\mathcal{X}|$ denoting its cardinality. Uppercase and lowercase letters, e.g., $x$ and $X$, represent scalars, lowercase boldface letters, e.g., $\boldsymbol{x}$, designate column vectors where $x_{i}$ indicates the $i$ th element, and uppercase boldface letters, e.g., $\boldsymbol{X}$, denote matrices where $X_{i, j}$ denotes the $(i, j)$ th element. The superscript $(\cdot)^{\mathrm{T}}$ stands for the transposition. Unless otherwise specified, vector and matrix inequalities are operated element-wise. Besides, $\mathbb{E}[\cdot]$ indicates the expectation.

\section{B. System Model}

Consider a single cell environment with $M^{\prime}$ C-UEs and $K^{\prime}$ V-UEs. The latter is counted in terms of transmitters. The corresponding sets are denoted by $\mathcal{M}^{\prime} \triangleq\left\{1,2, \ldots, M^{\prime}\right\}$ and $\mathcal{K}^{\prime} \triangleq\left\{1,2, \ldots, K^{\prime}\right\}$, respectively. The D2D underlay is only used by V-UEs that share the uplink radio resources. The uplink bandwidth is divided into $F$ RBs for each scheduling time unit. As traditional cellular users, orthogonal RB allocation applies to the C-UEs in the uplink, and RB allocation can be performed by any reasonable scheduling scheme. On the other hand, unlike existing works [5], [7], one C-UE's RBs may be shared by multiple V-UEs simultaneously, which brings the possibility of non-orthogonality among V-UE pairs. In addition, one V-UE may reuse the RBs of multiple C-UEs. Clearly, intra-cell interference may arise in the setup.

Fig. 1 illustrates intra-cell interference by dotted lines, where the C-UE $m^{\prime}$, the V-UE pairs $k^{\prime}$ and $l^{\prime}$ are using the same RB. Here, $h_{m^{\prime}}^{\prime}, h_{k^{\prime}}$, and $h_{l^{\prime}}$ are the effective channel power gains of the three desired transmissions, respectively, $g_{m^{\prime}, k^{\prime}}^{\prime}$ and $g_{m^{\prime}, l^{\prime}}^{\prime}$ denote the two interference channel power gains from the C-UE to the receivers of V-UE $k^{\prime}$ and $l^{\prime}$, and $g_{k^{\prime}}$ and $g_{l^{\prime}}$ are interfering channel power gains of the two V-UE transmissions to the C-UE's receiver (i.e., eNB). Moreover, $g_{k^{\prime}, l^{\prime}}$ and $g_{l^{\prime}, k^{\prime}}$ represent the cross-talk, interference channel power gains between the two V-UE pairs. We define $g_{k^{\prime}, k^{\prime}} \triangleq 0$ for all $k^{\prime} \in \mathcal{K}^{\prime}$. To perform RRM, the eNB needs CSI (at least to some degree) for all these involved links, where $h_{m^{\prime}}^{\prime}, g_{k^{\prime}}$, and $g_{l^{\prime}}$ can be measured at the eNB itself, but all the other links have to be measured by the corresponding V-UE receivers and then reported back to the eNB.

Due to signaling overhead, V-UEs should only feedback slowly varying CSI, including path loss and shadowing that have small variation over a few hundred milliseconds, to the eNB. For this reason, we argue that the eNB should only utilize slowly varying CSI and conduct long-term (e.g., a few hundred milliseconds) RRM for D2D-based V2X communications. Since path loss and shadowing are not greatly influenced by frequency in the considered bandwidth, we assume that the

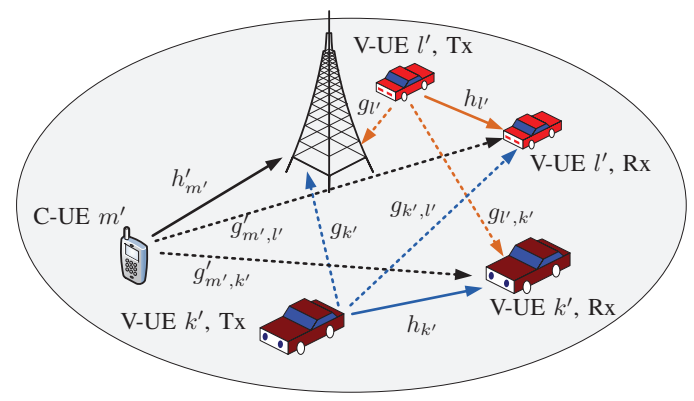

Figure 1. Interference between V2X and cellular communications.

CSI is frequency-invariant. Nevertheless, in Section V, we will also consider fast fading effects in simulations to evaluate the robustness of the proposed long-term RRM scheme.

\section{Requirements for V-UEs and C-UEs}

For safety-critical V2X services, there are stringent latency and reliability requirements, though high data rate is of less significance. Hence, the V-UEs' QoS requirements have to be modeled as strict constraints in our mathematical formulation. Due to the delay constraints as well as the limited bandwidth, the number of RBs allocated to each V-UE has to be bounded. Allocating $E_{k^{\prime}}^{\text {all }}$ RBs to the V-UE $k^{\prime}$, the outage probability, evaluated for a requirement of delivering $N_{k^{\prime}}$ bits, equals [11]

$$
p_{k^{\prime}}^{\text {out }} \triangleq \operatorname{Pr}\left\{\sum_{i=1}^{E_{k^{\prime}}^{\text {all }}} \rho \log _{2}\left(1+\gamma_{i}\right)<N_{k^{\prime}}\right\}
$$

where $\gamma_{i} \triangleq \bar{P}_{i}^{\mathrm{r}}\left|H_{i}\right|^{2} /\left(\sigma^{2}+\sum_{j=1}^{J} \bar{S}_{j, i}^{\mathrm{r}}\left|G_{j, i}\right|^{2}\right)$ is the instantaneous signal to interference plus noise ratio (SINR) of the RB $i$. Here, $J$ is the number of interference sources. $\bar{P}_{i}^{\mathrm{r}}$ and $\bar{S}_{j, i}^{\mathrm{r}}$ are the average received power from the desired and interfering users, respectively. $H_{i}$ and $G_{j, i}$ are random variables representing the fast fading effects of the corresponding desired and interference channels, respectively. $\sigma^{2}$ is the noise power and $\rho$ is the number of complex symbols per RB. Then, the reliability requirement is interpreted from the perspective of outage probability and can be expressed as [2]

$$
p_{k^{\prime}}^{\text {out }} \leq p_{\mathrm{o}}
$$

where $p_{\mathrm{o}}$ is the maximum tolerable outage probability.

We will replace (2) by a more strict constraint to account for that only slow varying CSI is assumed to be available to the eNB, To this end, we first upper-bound $p_{k^{\prime}}^{\text {out }}$ via Lemma 1.

Lemma 1. $p_{k^{\prime}}^{\text {out }}$ is upper-bounded by

$$
p_{k^{\prime}}^{\text {out }} \leq \operatorname{Pr}\left\{\sum_{i=1}^{E_{k^{\prime}}^{\text {all }}} \rho \log _{2}\left(1+\bar{\gamma}_{i}\left|H_{i}\right|^{2} \min \left\{\frac{1}{\left|G_{i}\right|_{\max }^{2}}, 1\right\}\right)<N_{k^{\prime}}\right\},
$$

where $\left|G_{i}\right|_{\max }^{2} \triangleq \max _{j=1,2, \ldots J}\left\{\left|G_{j, i}\right|^{2}\right\}$, and $\bar{\gamma}_{i} \triangleq \bar{P}_{i}^{\mathrm{r}} /\left(\sigma^{2}+\right.$ $\left.\sum_{j=1}^{J} \bar{S}_{j, i}^{\mathrm{r}}\right)$ requires only the slowly varying CSI. Note that $\bar{\gamma}_{i} \neq \mathbb{E}\left[\gamma_{i}\right]$. Also, the larger $J$ is, the looser the upper bound is. 
Proof: Due to the space limitation, a rigorous proof is not given here but will be presented in our future work.

Utilizing Lemma 1, we can constraint the upper bound in (3) to assure the original outage probability constraint in (2). Then, we further restrict the new outage probability requirement by means of the following two inequalities.

$$
\operatorname{Pr}\left\{\sum_{i=1}^{E_{k^{\prime}}^{\text {all }}} \rho \log _{2}\left(1+\bar{\gamma}_{k^{\prime}}^{\mathrm{T}}(J)\left|H_{i}\right|^{2} \min \left\{\frac{1}{\left|G_{i}\right|_{\max }^{2}}, 1\right\}\right)<N_{k^{\prime}}\right\} \leq p_{\mathrm{o}}
$$$$
\bar{\gamma}_{i} \geq \bar{\gamma}_{k^{\prime}}^{\mathrm{T}}(J), \quad \forall i=1,2, \ldots, E_{k^{\prime}}^{\mathrm{all}} .
$$

For the V-UE $k^{\prime}$ that shares a common RB with other $J$ UEs (including both C-UE and V-UEs), by deriving $\bar{\gamma}_{k^{\prime}}^{\mathrm{T}}(J)$ from (4) and requiring the actual $\bar{\gamma}_{i}$ of each used RB to be greater than or equal to $\bar{\gamma}_{k^{\prime}}^{\mathrm{T}}(J)$, we can guarantee the fulfillment of (2). Note that $\bar{\gamma}_{i}$ contains our optimization variables that will be formally defined later on. From now on, with a slight abuse of terminology, we refer to (5) as the SINR constraint under $J$ interference UEs.

For a given $\rho, N_{k^{\prime}}, p_{\mathrm{o}}$, and the probability density functions (pdfs) of $H_{i}$ as well as $G_{j, i}, \bar{\gamma}_{k^{\prime}}^{\mathrm{T}}(J)$ and $E_{k^{\prime}}^{\text {all }}$ can be treated as functions of each other. In this paper, we assume a fixed $E_{k^{\prime}}^{\text {all }}$ and then derive $\bar{\gamma}_{k^{\prime}}^{\mathrm{T}}(J)$ from $E_{k^{\prime}}^{\text {all }}$ for various values of $J$ by, e.g., Monte Carlo (MC) simulations.

Similar to [5], we reduce the two-dimensional RB allocation problem over both frequency and time into a sequence of onedimensional problems in the frequency domain. Toward this end, the requirements on latency and reliability become

$$
E_{k^{\prime}}=\left\lceil E_{k^{\prime}}^{\text {all }} / L_{\text {tol }}\right\rceil, \quad \bar{\gamma}_{i} \geq \bar{\gamma}_{k^{\prime}}^{\mathrm{T}}(J), \quad \forall i=1,2, \ldots, E_{k^{\prime}},
$$

where $L_{\text {tol }}$ is the latency constraint on V2X communication in terms of the number of scheduling time units, and $E_{k^{\prime}}$ is the number of RBs allocated to the V-UE $k^{\prime}$ during each scheduling time unit.

By taking the steps outlined thus far, we have transformed the original $\mathrm{V} 2 \mathrm{X}$ requirements on latency and reliability into constraints on $E_{k^{\prime}}$ and $\bar{\gamma}_{k^{\prime}}^{\mathrm{T}}(J)$, and the latter requires slow varying CSI only. In effect, for an arbitrary V-UE $k^{\prime}$ sharing RB with $J$ additional UEs, allocating $E_{k^{\prime}}$ RBs during each time unit and ensuring that for any of these RBs, say RB $i$, the actual $\bar{\gamma}_{i}$ is at least as large as $\bar{\gamma}_{k^{\prime}}^{\mathrm{T}}(J)$, the original latency and reliability requirement of the V-UE will be able to meet with some margin.

In comparison to V2X communications, most cellular data traffic types have much less strict latency requirement, and the system usually strives for high data rate under subject to some level of fairness. Therefore, the maximization of C-UEs' sum rate will be used as the optimization objective in our problem that will be formally stated in the next section.

To address the fairness among C-UEs, here we follow the proportional bandwidth fairness concept [12]. As a result, the number of RBs $E_{m^{\prime}}^{\prime}$ allocated to the C-UE $m^{\prime}$ during one scheduling time unit is given for all $m^{\prime} \in \mathcal{M}^{\prime}$ and $\sum_{m^{\prime}=1}^{M^{\prime}} E_{m^{\prime}}^{\prime}=F$.

\section{Problem Formulation}

In this section we detail the problem formulation that jointly deals with RRM for V-UEs and C-UEs. The performance objective is to maximize the C-UEs' sum rate with the aforementioned fairness consideration for the C-UE, subject to satisfying V-UEs' requirements on latency and reliability, i.e., the conditions defined by (6).

For notational convenience, we introduce the concepts of sub-users and extended user sets. Specifically, we split the VUE $k^{\prime}$ into $E_{k^{\prime}}$ sub-V-UEs for all $k^{\prime} \in \mathcal{K}^{\prime}$, and split the C-UE $m^{\prime}$ into $E_{m^{\prime}}^{\prime}$ sub-C-UEs for all $m^{\prime} \in \mathcal{M}^{\prime}$, where each sub-user corresponds to the allocation of one RB to the UE in question. Moreover, we define two extended user sets $\mathcal{K} \triangleq\{1,2, \ldots, K\}$ with $K=|\mathcal{K}|$ and $\mathcal{M} \triangleq\{1,2, \ldots, M\}$ with $M=|\mathcal{M}|$ for the sub-V-UEs and sub-C-UEs, respectively. To relate the original user sets and the extended user sets to each other, we define mapping $\hat{k}: \mathcal{K} \rightarrow \mathcal{K}^{\prime}$ such that $k^{\prime}=\hat{k}(k)$ is the V-UE to which the sub-V-UE $k$ belongs. Similarly, mapping $\hat{m}: \mathcal{M} \rightarrow \mathcal{M}^{\prime}$ such that $m^{\prime}=\hat{m}(m)$ is the C-UE to which the sub-C-UE $m$ belongs.

Based on the above definitions, the joint RRM problem for V-UEs is formalized below.

$$
\max \sum_{m=1}^{M} \log _{2}\left(1+\frac{S_{m} h_{\hat{m}(m)}^{\prime}}{\sigma^{2}+\sum_{k=1}^{K} P_{m, k} g_{\hat{k}(k)}}\right)
$$

subject to:

$$
\begin{aligned}
& x_{m, k} \in\{0,1\}, \quad P_{m, k} \leq P^{\max } x_{m, k} \quad \forall m, k \\
& 0 \leq P_{m, k}, \sum_{m=1}^{M} \sum_{k, \hat{k}(k)=k^{\prime}} P_{m, k} \leq P^{\max }, \quad \forall k^{\prime} \\
& 0 \leq S_{m}, \quad \sum_{m, \hat{m}(m)=m^{\prime}} S_{m} \leq S^{\max }, \quad \forall m^{\prime} \\
& \sum_{m=1}^{M} x_{m, k}=1, \quad \forall k \\
& \sum_{k=1}^{K} x_{m, k}=J_{m}, \quad J_{m} \in \mathcal{K}^{\prime}, \quad \forall m \\
& \sigma^{2}+S_{m} g_{\hat{m}(m), \hat{k}(k)}^{\prime}+\sum_{l=1, l \neq k}^{K} P_{m, l} g_{\hat{k}(l), \hat{k}(k)} \\
& \geq x_{m, k} \bar{\gamma}_{\hat{k}(k)}^{\mathrm{T}}\left(J_{m}\right), \quad \forall m, k
\end{aligned}
$$

where $k \in \mathcal{K}, m \in \mathcal{M}, k^{\prime} \in \mathcal{K}^{\prime}, m^{\prime} \in \mathcal{M}^{\prime}$. In the formulation, $x_{m, k}$ is a binary variable that equals 1 if the sub-V-UE $k$ is sharing the same RB with the sub-C-UE $m$ and 0 otherwise, $S_{m}$ is the transmit power of the sub-C-UE $m$, and $P_{m, k}$ is the transmit power of the sub-V-UE $k$ on the RB shared by the sub-C-UE $m$. If this sharing does not take place, i.e., $x_{m, k}=0$, then $P_{m, k}$ must be 0 as well; this is implied by constraint (7b). Constraints $(7 \mathrm{c})$ and $(7 \mathrm{~d})$ state the max transmit power for each V-UE and C-UE, respectively. Equation (7e) ensures that each sub-V-UE is paired with exact one sub-C-UE, and equation (7f) implies that $J_{m}$ sub-V-UEs are sharing the same RB with the sub-C-UE $m$. Last but not least, constraint $(7 \mathrm{~g})$ formulates the SINR requirement for each sub-V-UE, where the left-hand side is interpreted as $\bar{\gamma}_{k}$. It should also be remarked that, in the formulation, RB sharing among multiple sub-V-UEs is admitted, in contrast to the setup 
in [5] that only allows V-UEs to use orthogonal RBs.

In problem (7), the input consists of $M^{\prime}, K^{\prime}, E_{m^{\prime}}^{\prime}, E_{k^{\prime}}, \sigma^{2}$, $P^{\max }, S^{\max }, h_{m^{\prime}}^{\prime}, g_{k^{\prime}}, h_{k^{\prime}}, g_{m^{\prime}, k^{\prime}}^{\prime}, g_{k^{\prime}, l^{\prime}}$, and $\bar{\gamma}_{k^{\prime}}^{\mathrm{T}}(J)$ for all $J \in \mathcal{K}^{\prime}$. The output, represented by the optimization variables, is given by $x_{m, k}, P_{m, k}$, and $S_{m}$ for all $k \in \mathcal{K}$ and $m \in \mathcal{M}$.

Theorem 1. The $R B$ sharing and power allocation problem (7) for D2D-based V2X communication is NP-hard.

Proof: Due to the space limitation, the proof is not given here but to be published elsewhere. The basic idea is to reduce the partition problem, which is known to be NP-complete, to problem (7).

In our reduction proof for Theorem 1, the sum-power limits (7c) and (7d) are not in effect. Hence we obtain the following corollary.

Corollary 1. The RB sharing and power allocation problem (7) remains NP-hard even if maximum sum- power constraints (7c) and (7d) are relaxed.

Due to the NP-hardness of problem (7), we cannot expect the existence of an algorithm that is both time-efficient and guarantees global optimality, unless $\mathrm{P}=\mathrm{NP}$. Therefore, we resort to heuristic algorithms for problem solution.

\section{The Proposed RBSPA Algorithm}

In this section we propose a heuristic RBSPA scheme to solve problem (7). To this end, we will first in Section IV-A introduce the theoretical motivation of the proposed approach, utilizing the Perron-Frobenius theory [13]. Then we will derive the RBSPA scheme that consists of two stages that are detailed in Sections IV-B and IV-C, respectively. In the first stage, the max power constraints for both V-UEs and C-UEs are relaxed, and the eNB assigns RB to each sub-V-UE according to a specific metric in a sequential manner. The metric is driven by the Perron-Frobenius theory. In the second stage, based on the RB sharing outcome of stage one, the eNB optimally adjusts the transmit power for each V-UE and C-UE, taking the sum power constraints into account.

\section{A. The Underlying Theory}

Consider $N$ UE pairs using the same resource for data transmission, where each UE pair has a received SINR constraint $\gamma_{i}^{\mathrm{T}}$, i.e.,

$$
\gamma_{i}(\boldsymbol{p}) \triangleq \frac{w_{i, i} p_{i}}{\sum_{j \neq i} w_{j, i} p_{j}+\sigma_{i}^{2}} \geq \gamma_{i}^{\mathrm{T}}, \quad i=1,2, \ldots, N
$$

where $\gamma_{i}(\boldsymbol{p})$ is defined as the received SINR for the receiver $i, w_{j, i}$ denotes the channel power gain from the transmitter $j$ to the receiver $i, p_{i}$ is the transmit power of the transmitter $i$, and $\sigma_{i}^{2}$ is the noise power at the receiver $i$. By defining

$$
\Omega_{i, j} \triangleq\left\{\begin{array}{lr}
\gamma_{i}^{\mathrm{T}} w_{j, i} / w_{i, i} & \text { if } i \neq j \\
0 & \text { otherwise, }
\end{array}\right.
$$

and $q_{i} \triangleq \gamma_{i}^{\mathrm{T}} \sigma_{i}^{2} / w_{i, i}$, the matrix form of (8) is $(\boldsymbol{I}-\boldsymbol{\Omega}) \boldsymbol{p} \geq \boldsymbol{q}$.

For convenience, we name $\Omega \in \mathbb{R}^{N \times N}$ the inherent constraint matrix associated to the model in (8). Note this matrix is non-negative. To ease the presentation, here we assume $\Omega$ is irreducible $^{1}$. The assumption of irreducible $\Omega$ comes with no

\footnotetext{
${ }^{1}$ Replacing all non-zero entries in a matrix by one, and viewing the matrix as the adjacency matrix of a directed graph, the matrix is called irreducible if and only if such directed graph is strongly connected.
}

loss of generality, because otherwise the problem decomposes into independent and smaller parts, each being associated with an irreducible inherent constraint matrix [14].

We present two lemmas which play crucial roles in deriving the RBSPA algorithm.

Lemma 2. Assuming the inherent constraint matrix $\Omega$ associated with (8) irreducible, we have

$$
\rho(\boldsymbol{\Omega})=\inf _{\boldsymbol{p}>\mathbf{0}} \max _{i=1, \ldots, N} \frac{\gamma_{i}^{\mathrm{T}}}{\gamma_{i}(\boldsymbol{p})},
$$

where $\rho(\boldsymbol{\Omega})$ denotes the spectral radius of matrix $\boldsymbol{\Omega}$.

Proof: Firstly, note that

$$
\begin{aligned}
& \inf _{\boldsymbol{p}>\mathbf{0}} \max _{i=1, \ldots, N} \frac{\gamma_{i}^{\mathrm{T}}}{\gamma_{i}(\boldsymbol{p})}=\inf _{\boldsymbol{p}>\mathbf{0}} \max _{i=1, \ldots, N} \frac{\sum_{j=1}^{N} \Omega_{i, j} p_{j}+q_{i}}{p_{i}} \\
& =\inf _{\substack{\alpha>0, \tilde{\boldsymbol{p}}>\mathbf{0} \\
\tilde{\boldsymbol{p}}^{\top} \tilde{\boldsymbol{p}}=1}} \max _{i=1, \ldots, N} \sum_{j=1}^{N} \Omega_{i, j} \frac{\alpha \tilde{p}_{j}}{\alpha \tilde{p}_{i}}+\frac{q_{i}}{\alpha \tilde{p}_{i}} \\
& =\inf _{\tilde{\boldsymbol{p}}^{\mathrm{T}} \tilde{\boldsymbol{p}}=1, \tilde{\boldsymbol{p}}>\mathbf{0}} \max _{i=1, \ldots, N} \sum_{j=1}^{N} \Omega_{i, j} \tilde{p}_{j} / \tilde{p}_{i} \\
& =\inf _{\boldsymbol{p}>\mathbf{0}} \max _{i=1, \ldots, N} \sum_{j=1}^{N} \Omega_{i, j} p_{j} / p_{i},
\end{aligned}
$$

where (11) follows by the definitions of $\Omega_{i, j}$ and $q_{i} ;(12)$ is true due to the fact that any $\boldsymbol{p}>\mathbf{0}$ can be expressed as $\boldsymbol{p}=\alpha \tilde{\boldsymbol{p}}$ for certain $\alpha>0$ and $\tilde{\boldsymbol{p}}>\mathbf{0}$ with $\tilde{\boldsymbol{p}}^{\top} \tilde{\boldsymbol{p}}=1$; (13) follows since the value of $\alpha \tilde{p}_{j} /\left(\alpha \tilde{p}_{i}\right)$ is independent of $\alpha$, but $q_{i} /\left(\alpha \tilde{p}_{i}\right)$ decreases to 0 when $\alpha \rightarrow+\infty$; and (14) is true because scaling $\tilde{p}$ does not change the value of the objective function in (13).

Furthermore, from the Theorem A.47 in [15], we know that

$$
\rho(\boldsymbol{\Omega})=\inf _{\boldsymbol{p}>\mathbf{0}} \max _{i=1, \ldots, N} \sum_{j=1}^{N} \Omega_{i, j} p_{j} / p_{i},
$$

which concludes the proof.

Lemma 3. For $(\boldsymbol{I}-\boldsymbol{\Omega}) \boldsymbol{p} \geq \boldsymbol{q}$, a necessary and sufficient condition to have a positive solution $\boldsymbol{p}$ for every positive vector $\boldsymbol{q}$ is that $(\boldsymbol{I}-\boldsymbol{\Omega})^{-1}$ is nonnegative for $\boldsymbol{\Omega} \geq \mathbf{0}$. Moreover, for any $\boldsymbol{\Omega} \geq \mathbf{0},(\boldsymbol{I}-\boldsymbol{\Omega})^{-1} \geq \mathbf{0}$ if and only if $\rho(\boldsymbol{\Omega})<1$.

Proof: See [13].

\section{B. RB Sharing}

In the first stage of the RBSPA algorithm, maximum power limits for both V-UEs and C-UEs are relaxed. Now the problem of RB sharing becomes to pair the sub-V-UEs with sub-C-UEs in a way such that the sub-C-UEs' sum rate is maximized and the sub-V-UEs' SINR constraints are met. Due to the NP-hardness stated in Corollary 1, we propose a heuristic RB sharing scheme that associates each sub-V-UE with a sub-C-UE in a sequential fashion.

Assume that sub-V-UEs $\{1, \ldots, k-1\}$ have been already paired with sub-C-UEs, and let us focus on the sub-V-UE $k$ and find the most appropriate sub-C-UE for RB sharing. The decision has to account for two aspects: 1) The SINR constraints of the $k$ sub-V-UEs $\{1, \ldots, k\}$ should be satisfied (unless this is infeasible), and 2) the sub-C-UE that has the 
best rate when sharing its RB should be selected. However, the challenge here is that power is not set at this stage, prohibiting the evaluation of the sub-V-UEs' and the sub-C-UEs' rates. Therefore, in the following, we will design a metric that is independent of the power parameter for the pairing process, and justify its rationale.

For all $m \in \mathcal{M}$, denote by $\mathcal{K}_{m}^{(k-1)}$ the indices of the sub$\mathrm{V}$-UEs in $\{1, \ldots, k-1\}$ such that RB sharing takes places with the sub-C-UE $m$, i.e., $\mathcal{K}_{m}^{(k-1)} \triangleq\{l \mid l \in\{1, \ldots, k-$ $\left.1\}, x_{m, l}=1\right\}$. If the sub-V-UE $k$ would also use the subC-UE $m$ 's RB, the SINR constraints $(7 \mathrm{~g})$ for the sub-V-UEs in $\tilde{\mathcal{K}}_{m}^{(k)} \triangleq \mathcal{K}_{m}^{(k-1)} \cup\{k\}$ are

$$
\begin{aligned}
& P_{m, l}-\sum_{l^{\prime} \in \tilde{\mathcal{K}}_{m}^{(k)}, l^{\prime} \neq l} P_{m, l^{\prime}} g_{\hat{k}\left(l^{\prime}\right), \hat{k}(l)} \bar{\gamma}_{\hat{k}(l)}^{\mathrm{T}}\left(J_{m}^{(k)}\right) / h_{\hat{k}(l)} \\
& \geq S_{m} g_{\hat{m}(m), \hat{k}(l)}^{\prime} \bar{\gamma}_{\hat{k}(l)}^{\mathrm{T}}\left(J_{m}^{(k)}\right) / h_{\hat{k}(l)}+\sigma^{2} \bar{\gamma}_{\hat{k}(l)}^{\mathrm{T}}\left(J_{m}^{(k)}\right) / h_{\hat{k}(l)},
\end{aligned}
$$

for all $l \in \tilde{\mathcal{K}}_{m}^{(k)}$, where $J_{m}^{(k)} \triangleq\left|\tilde{\mathcal{K}}_{m}^{(k)}\right|$. The inherent constraint matrix $\boldsymbol{\Omega}^{(k, m)} \in \mathbb{R}_{m}^{J_{m}^{(k)} \times J_{m}^{(k)}}$ associated with the inequality system (16) can be easily calculated. According to Lemma 3, when the sub-V-UE $k$ is paired with the sub-C-UE $m$, it is possible to satisfy the SINR constraints (16) if and only if $\rho\left(\boldsymbol{\Omega}^{(k, m)}\right)<1$.

Checking $\rho\left(\boldsymbol{\Omega}^{(k, m)}\right)$ for all $m \in \mathcal{M}$ yields the subset $\tilde{\mathcal{M}}$ containing the feasible sub-C-UEs for paring with the sub-V-UE $k$. The next step is to choose the sub-C-UE in $\tilde{\mathcal{M}}$ from the rate perspective. Even though rate cannot be exactly evaluated without setting the power, we can utilize the following observation to design a reasonable metric for choosing sub-C-UE.

Observation 1. From Lemma 2, the spectral radius of a set of SINR inequalities is closely connected to the attainable SINR levels and hence rates. Thus, loosely speaking, the smaller the spectral radius is, the better the attainable SINR of the sub$C$-UE, for which the maximum of the inner problem in (10) is achieved.

To utilize the observation, for each sub-C-UE $m \in \mathcal{M}$, we associate with a virtual SINR constraint $\beta_{m}^{\mathrm{T}}$, where $\beta_{m}^{\mathrm{T}} \triangleq$ $S^{\max } h_{\hat{m}(m)}^{\prime} / \sigma^{2}$. The reason for the choice of $\beta_{m}^{\mathrm{T}}$ is that, in line with our goal of maximizing rate for sub-C-UE and according to Observation 1 and Lemma 2, the maximum over $i \in\{1, \ldots, N\}$ in (10) may be reached at the sub-C-UE $m$ when considering the inherent constraint matrix of these selected SINR thresholds. By considering sub-C-UE $m$ as well as all sub-V-UEs $\tilde{\mathcal{K}}_{m}^{(k)}$, the SINR constraint system becomes

$$
\left\{\begin{array}{c}
(16) \\
S_{m}-\sum_{l \in \tilde{\mathcal{K}}_{m}^{(k)}} P_{m, l} g_{\hat{k}(l)} \beta_{m}^{\mathrm{T}} / h_{\hat{m}(m)}^{\prime} \geq \sigma^{2} \beta_{m}^{\mathrm{T}} / h_{\hat{m}(m)}^{\prime}
\end{array}\right\}
$$

for all $l \in \tilde{\mathcal{K}}_{m}^{(k)}$; the corresponding inherent constraint matrix $\tilde{\mathbf{\Omega}}^{(k, m)} \in \mathbb{R}^{\left(J_{m}^{(k)}+1\right) \times\left(J_{m}^{(k)}+1\right)}$ can be easily obtained. Then, we propose to assign the sub-V-UE $k$ with the sub-C-UE $m$ 's RB where $m=\arg \min _{m \in \tilde{\mathcal{M}}} \rho\left(\tilde{\boldsymbol{\Omega}}^{(k, m)}\right)$.

The steps the proposed RB sharing scheme are presented in Algorithm 1.

\footnotetext{
${ }^{2}$ Since the bound proposed in Lemma 1 becomes looser with the number of interference sources $J$, in the algorithm we restrict the number of V-UEs that may share the same RB to $J_{\max }$.
}

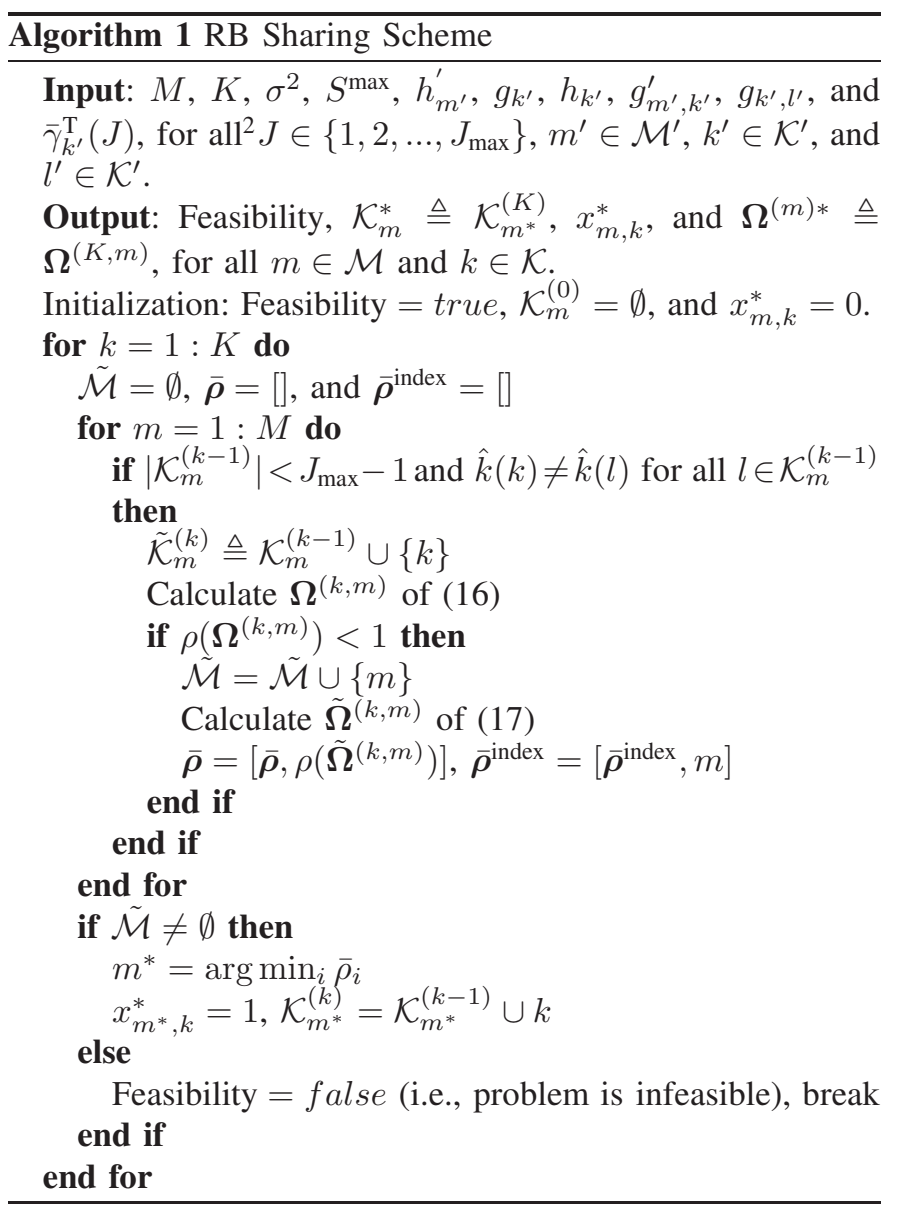

\section{Power Allocation}

If the problem is reported feasible by Algorithm 1, i.e., Feasibility $=$ true, the second stage of the proposed RBSPA scheme is to allocate power based on the RB allocation. In case Feasibility $=$ false, the problem is considered infeasible. This infeasibility can be caused by either the problem data or the limitation of the algorithm.

Assuming feasibility in RB allocation, the power allocation problem (PAP) is formulated similarly to (7), by replacing each $x_{m, k}$ with $x_{m, k}^{*}$ obtained from Algorithm 1 . Now the optimization variables are $S_{m}$ and $P_{m, k}$ for all $m \in \mathcal{M}$ and $k \in \mathcal{K}_{m}$. Since the objective function is not concave with respect to $P_{m, k}$, the PAP is not convex. Nevertheless, we can note that the objective function is monotonically nonincreasing in $P_{m, k}$, and thus at the maximum, equality must hold for $(7 \mathrm{~g})$ for sub-V-UEs' SINR, with the matrix form

$$
\left(\boldsymbol{I}-\boldsymbol{\Omega}^{(m) *}\right) \boldsymbol{p}_{m}=\boldsymbol{b}_{m}, \quad \forall m \in \mathcal{M},
$$

where $\boldsymbol{p}_{m}$ stacks $P_{m, k}$ and $\boldsymbol{b}_{m}$ stacks $\sigma^{2} \bar{\gamma}_{\hat{k}(k)}^{\mathrm{T}}\left(J_{m}^{*}\right)+$ $S_{m} g_{\hat{m}(m), \hat{k}(k)}^{\prime} \bar{\gamma}_{\hat{k}(k)}^{\mathrm{T}}\left(J_{m}^{*}\right)$ for all $k \in \mathcal{K}_{m}^{*}$ with $J_{m}^{*}=\left|\mathcal{K}_{m}^{*}\right|$. The order of the sub-V-UE indices in $\boldsymbol{p}_{m}$ and $\boldsymbol{b}_{m}$ is assumed to be consistent with that in $\boldsymbol{\Omega}^{(m) *}$.

Solving (18), we obtain $\boldsymbol{p}_{m}=\left(\boldsymbol{I}-\boldsymbol{\Omega}^{(m) *}\right)^{-1} \boldsymbol{b}_{m}$ for all $m \in \mathcal{M}$. Since the PAP is feasible without considering maximum power constraints, we have $\left(\boldsymbol{I}-\boldsymbol{\Omega}^{(m) *}\right)^{-1} \geq \mathbf{0}$ from Lemma 3. Therefore, for all $k \in \mathcal{K}_{m}^{*}, P_{m, k}$ can be expressed 
as an affine function of $S_{m}$ with nonnegative slope and offset. Using the optimal solution of $\boldsymbol{p}_{m}$, we can eliminate $P_{m, k}$ from the PAP. Then, the remaining problem is transformed into an equivalent convex optimization model, which can be solved optimally by an interior point method. The convexity can be shown through verifying the positive semidefinite property of the corresponding Hessian matrix, which is resulted from the nonnegative slope and offset analyzed above.

\section{Performance Evaluation}

We use a single cell outdoor system as the test scenario. In particular, we consider test case (TC) 2 defined by the METIS project [16]. Details of the system parameters and channel models are presented in [5].

\section{A. Performance Metrics and RRM Schemes}

We base our evaluation on three metrics:

- C-UEs' sum rate when the fast fading effect is disregarded (i.e., value of the objective in (7));

- cumulative distribution function (CDF) of the C-UEs' sum rate;

- CDF of one V-UE's transmitted bits within $5 \mathrm{~ms}$, i.e., the left-hand side of the inner inequality in (1).

Note that the last two metrics are evaluated with consideration of fast fading in the simulations.

The proposed RBSPA scheme is compared with the following baseline methods.

1) The two-stage SRBP algorithm in [5] that consists in $\mathrm{RB}$ allocation and power control, where RB allocation of $\mathrm{V}$ UEs is restricted to be orthogonal. For a given initial power, maximum weight matching (MWM) for bipartite graphs is used to achieve the optimal RB sharing.

2) SRBP-Ext, which is a simple extension of the SRBP scheme to nonorthogonal V-UEs, by performing MWM for each V-UE sequentially. Here the purpose of the extension is for the traffic load which is defined as the number of V-UEs, but not for the C-UE's rate performance.

3) Global optimal solution to problem (7) by the exhausitive search over all the RB sharing possibilities. Due to its exponentially increased complexity, we consider $F=4$.

\section{B. Simulation Results}

Based on the requirements given by METIS [2], we have $N_{k^{\prime}}=12800$ bits, $p_{0}=10^{-5}$ (i.e., a transmission reliability of $99.999 \%$ ), and $L_{\text {tol }}=10$ (i.e., a latency requirement of $5 \mathrm{~ms})$. Here we consider $E_{k^{\prime}}$ equal to 2 or 5. As results, by the analysis in Section II-C, some possible values of $\left\{E_{k^{\prime}}, \bar{\gamma}_{k^{\prime}}^{\mathrm{T}}[\mathrm{dB}], J\right\}$ are $\{2,32.6,1\}$, $\{2,33.4,2\}, \quad\{2,34.0,3\}, \quad\{2,34.5,4\}, \quad\{2,34.9,5\}$, $\{5,14.9,1\}, \quad\{5,15.7,2\}, \quad\{5,16.3,3\}, \quad\{5,16.7,4\}, \quad$ and $\{5,17.1,5\}$.

Fig. 2 compares the C-UEs' sum rates of the RRM schemes when $F=4$ and shows the performance gap in respect of the global optimum. Recall that the values shown in the labels are based on the long-term CSI, whereas the CDF curves represent results for which fast fading effects are accounted for. By comparing the values to the CDFs, it can be seen that even though the RRM decisions are based on long-term CSI, the results remain reasonably accurate for realistic channel conditions with fast fading. Comparing the RRM schemes, the

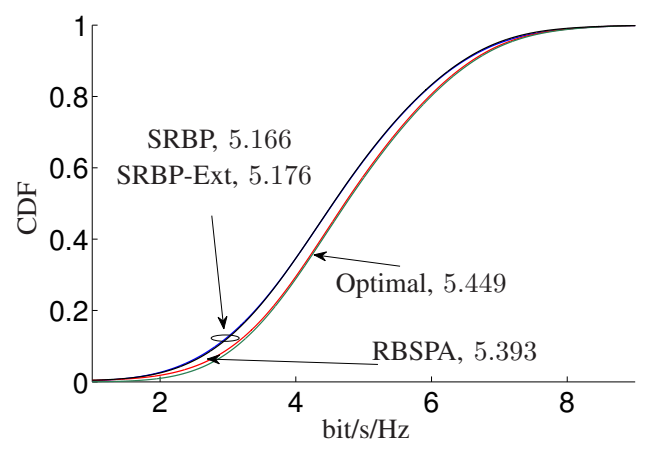

Figure 2. Sum rate of the C-UEs with $F=4, M^{\prime}=2, K^{\prime}=2, E_{m^{\prime}}^{\prime}=2$, $E_{k^{\prime}}=2$, and $J_{\max }=2$.

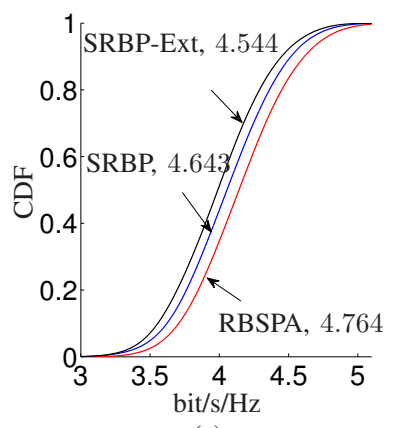

(a)

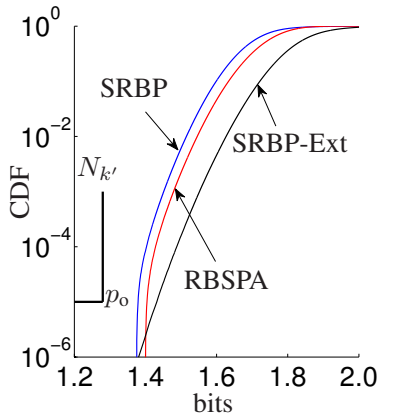

(b)
Figure 3. $F=100, M^{\prime}=25, K^{\prime}=50, E_{m^{\prime}}^{\prime}=4, E_{k^{\prime}}=2$, and $J_{\max }=5$. (a) Sum rate of C-UEs. (b) Transmitted bits within $5 \mathrm{~ms}$ for each V-UE.

performance difference of the proposed RBSPA to optimum is very small. On the other hand, SRBP and SRBP-Ext exhibit worse sum rates.

Now consider more realistic scenarios with $F=100$. We define the traffic load as the number of V-UEs. For a low load situation, i.e., $K^{\prime}=10$, Fig. 3(a) and Fig. 3(b) illustrate the performances of C-UEs and V-UEs respectively.

In Fig. 3(a), the CDFs of the C-UEs' sum rates are presented. By comparing the numbers in the labels and the CDF curves, it is again evident that, even though the RRM schemes use long-term CSI, lead to valid results even when fast fading effects are present. Moreover, compared to SRBP and SRBP-Ext, the proposed RBSPA scheme exhibits superiority. Fig. 3(b) depicts the CDFs of the transmitted bits within 5 ms for one V-UE. It can be seen that the outage probability constraint, which represents the QoS requirements of the VUEs, is fulfilled for all the three schemes. RBSPA and SRBPExt give higher number of bits than SRBP, because allowing non-orthogonality gives rise to increased SINR value in $(7 \mathrm{~g})$ and better rate (and here, more bits) for the V-UEs. SRBP-Ext achieves slightly higher number of bits than RBSPA as the former allocates more V-UEs on the same RB. This, in fact, illustrates a disadvantage of SRBP-Ext since the increased VUE's rate is at the price of the C-UE's rate (see Fig. 3(a)), which is the objective in (7).

Next, the C-UEs' sum rates are plotted in Fig. 4(a) and Fig. 4(b) with respect to various load levels of the of vehicular communications (i.e., values of $K^{\prime}$ ) for $E_{k^{\prime}}=2$ and $E_{k^{\prime}}=5$. 


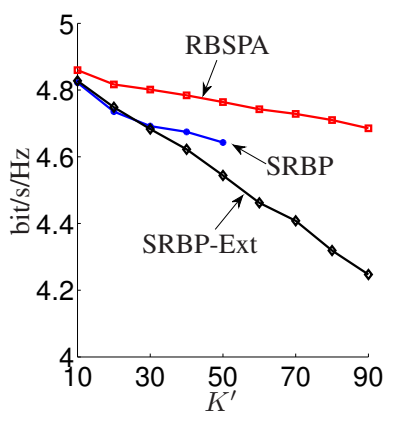

(a)

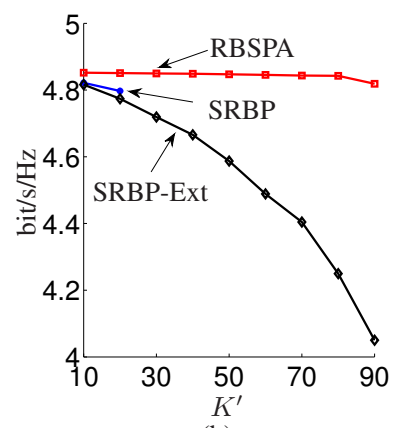

(b)
Figure 4. Sum rate of C-UEs versus number of V-UEs $\left(K^{\prime}\right)$ with $F=100$, $M^{\prime}=25, E_{m^{\prime}}^{\prime}=4$, and $J_{\max }=5$. (a) $E_{k^{\prime}}=2$. (b) $E_{k^{\prime}}=5$.

By SRBP, the maximum supported numbers of V-UEs are 50 and 20 for $E_{k^{\prime}}=2$ and $E_{k^{\prime}}=5$, respectively; these values represent the limit of orthogonal resource allocation. By allowing non-orthogonality, RBSPA and SRBP-Ext enables vehicular communications with much higher loads. Note that, within the feasible range of SRBP, SRBP outperforms SRBPExt as $K^{\prime}$ increases. The reason is that, even though allowing non-orthogonality among V-UEs may bring less interference to C-UEs by spatial reuse, there are two potential drawbacks. First, the values in the SINR constraint $(7 \mathrm{~g})$ becomes larger when more V-UEs are sharing the same RB. As a result, the transmit power of the V-UEs will be higher, leading to more interference to the RB-shared C-UE. Second, in SRBP, MWM is used for the RB sharing stage. As the corresponding problem become NP-hard for nonorthogonal RB allocation, sequential MWM is deployed by SRBP-Ext, and the performance gap between the optimum and sequential matching becomes larger with increased $K^{\prime}$. However, it should be emphasized that the most important advantage of allowing non-orthogonality is to support a significantly higher number of V-UEs. Last but not least, RBSPA provides the best performance for all the considered scenarios. Compared to SRBP-Ext, RBSPA exhibits not only improved rate, but also robustness to the number of V-UEs and the superiority of RBSPA becomes more apparent when $K^{\prime}$ is larger.

When it comes to complexity, the difference of the three schemes lies in the RB sharing stage. Based on the properties of MWM [5], the computational complexities of the RB sharing procedures in the SRBP and SRBP-Ext are upper-bounded by $O\left(F^{3}\right)$ and $O\left(K^{\prime} F^{3}\right)$, respectively. On the other hand, the most complex part of the RBSPA scheme is on the calculation of spectral radius which has the complexity of $O\left(N^{3}\right)$ for a $N \times N$ matrix. Correspondingly, the computational complexity of the RB sharing stage in RBSPA is about $O\left(K^{\prime} F N^{3}\right)$ with $N \leq 5$, which gives much lower complexity compared to the SRPB-Ext scheme.

\section{CONCLUSION}

Adopting direct D2D links is a promising enabler for safetycritical V2X communications, though this requires the development of application-tailored RRM schemes. In this paper, we have presented an approach to transform the strict latency and reliability requirements of $\mathrm{V} 2 \mathrm{X}$ communications into mathematical constraints that can be computed using slowly varying CSI only. Utilizing the mathematical constraints, the RRM process is then formulated as an optimization problem, where V-UEs are allowed to share RBs. By allowing this non-orthogonality among the V-UEs, the supported number of V-UEs can be significantly increased. Moreover, we have proposed the RBSPA scheme to solve the optimization problem. As demonstrated by the simulation results, the proposed scheme leads to not only improved cellular sum rate performance but also robustness to the number of V-UEs, which promotes its application in practice.

\section{REFERENCES}

[1] A. Khelil and D. Soldani, "On the suitability of device-to-device communications for road traffic safety," in IEEE World Forum on Internet of Things (WF-IoT), 2014.

[2] "Scenarios, requirements and KPIs for 5G mobile and wireless system," ICT-317669-METIS/D1.1, METIS deliverable D1.1, Apr. 2013. [Online]. Available: https://www.metis2020.com/documents/deliverables/

[3] G. Fodor, E. Dahlman, G. Mildh, S. Parkvall, N. Reider, G. Miklos, and Z. Turanyi, "Design aspects of network assisted device-to-device communications," IEEE Trans. Comm. Mag., vol. 50, no. 3, pp. 170$177,2012$.

[4] M. Botsov, M. Klugel, W. Kellerer, and P. Fertl, "Location dependent resource allocation for mobile device-to-device communications," in IEEE WCNC, Apr. 2014.

[5] W. Sun, E. G. Ström, F. Brännström, Y. Sui, and K. C. Sou, "D2Dbased V2V communications with latency and reliability constraints," in IEEE GLOBECOM Workshops, Dec. 2014. [Online]. Available: http://arxiv.org/abs/1501.02973

[6] A. Asadi, Q. Wang, and V. Mancuso, "A survey on device-to-device communcation in cellular networks," IEEE Communications Surveys \& Tutorials, no. 99, Apr. 2014.

[7] D. Feng, L. Lu, Y. Yuan-Wu, G. Y. Li, G. Feng, and S. Li, "Device-todevice communications underlaying cellular networks," IEEE Transactions on Communications, vol. 61, no. 8, pp. 3541-3551, Aug. 2013.

[8] R. Zhang, X. Cheng, L. Yang, and B. Jiao, "Interference-aware graph based resource sharing for device-to-device communications underlaying cellular networks," in IEEE Wireless Communications and Networking Conference (WCNC), Apr. 2013, pp. 140-145.

[9] D. H. Lee, K. W. Choi, W. S. Jeon, and D. G. Jeong, "Two-stage semidistributed resource management for device-to-device communication in cellular networks," IEEE Trans. Wireless Commun., vol. 13, no. 4, pp. 1908-1920, Apr. 2014.

[10] P. Phunchongharn, E. Hossain, and D. I. Kim, "Resource allocation for device-to-device communications underlaying LTE-advanced networks," IEEE Trans. Wireless Commun., vol. 20, no. 4, pp. 91-100, Aug. 2013.

[11] G. Caire, G. Taricco, and E. Biglieri, "Optimum power control over fading channels," IEEE Trans. Inf. Theory, vol. 45, no. 5, pp. 14681489, Jul. 1999.

[12] S. Sadr, A. Anpalagan, and K. Raahemifar, "A novel subcarrier allocation algorithm for multiuser OFDM system with fairness: User's perspective," in IEEE Vehicular Technology Conference (VTC), 2007, pp. 1772-1776.

[13] E. Seneta, Non-Negative Matrices and Markov Chains. Springer Verlag, 2006.

[14] S. Stanczak, H. Boche, and M. Wiczanowski, "Towards a better understanding of medium access control for multiuser beamforming systems," in IEEE Wireless Communications and Networking Conference (WCNC), Mar. 2005.

[15] S. Stanczak, M. Wiczanowski, and H. Boche, Fundamentals of Resource Allocation in Wireless Networks. Springer Publishing Company, 2009.

[16] "Simulation guidelines," ICT-317669-METIS/D6.1, METIS deliverable D6.1, Oct. 2013. [Online]. Available: https://www.metis2020.com/documents/deliverables/ 